\title{
Opinião
}

\section{PERCEPÇÃO DOS ENFERMEIROS QUANTO À PARTICIPAÇÃO DO ACOMPANHANTE DURANTE A INTERNAÇÃO HOSPITALAR DE UM USUÁRIO DO SISTEMA ÚNICO DE SAÚDE (SUS)}

\author{
Juliana Saraiva de Alencar (1) \\ Lorena Saraiva de Alencar(2) \\ Patrícia Luciany Almeida Macêdo da Silva (3) \\ Josberto Calixto Pereira (4)
}

\begin{abstract}
Resumo
Este estudo teve como objetivo conhecer a percepção dos Enfermeiros de um hospital filantrópico, na cidade do Crato-Ce, quanto à participação do acompanhante durante a internação hospitalar de um usuário do Sistema Único de Saúde. Percorreu a trajetória metodológica da pesquisa qualitativa/descritiva. Foi desenvolvida com Enfermeiros que atenderam aos critérios de inclusão da pesquisa: ser enfermeiro da instituição, prestar assistência direta aos pacientes usuários do S.U.S. e anuência para participar do estudo. A coleta de dados foi realizada no primeiro semestre de 2011, teve como instrumento de pesquisa um formulário semi-estruturado, para a sua aplicação foram considerados o aceite do termo de consentimento e a Resolução 196/96. A análise e discussão dos dados foram dispostos em categorias de estudo. Os resultados apresentados consideram relevante a presença do familiar junto ao paciente, por estes serem fundamentais no auxílio ao profissional durante o desenvolvimento de procedimentos, como também atuam no apoio psicossocial para aceitação do novo ambiente, das normas e rotinas da instituição hospitalar. Diante dos resultados apresentados e confrontando com o apresentado na literatura, é importante que seja realizado um trabalho de conscientização dos profissionais quanto à importância desse novo membro que passa a estar presente nas instituições de saúde, bem como desenvolver políticas em que as definições do papel do cuidador e do profissional sejam esclarecidas, reduzindo ou eliminando conflitos na relação acompanhante e profissional durante o período de hospitalização do familiar, de forma que não se tenha interferências negativas no processo do cuidar.
\end{abstract}

Palavras-chave: Hospitalização. Acompanhante. Enfermagem.

\section{Introdução}

Com o surgimento dos hospitais e os avanços tecnológicos sob a ótica biologicista, a visão do processo de adoecer é modificada e passa a ser reduzida à perspectiva do corpo como uma máquina defeituosa, onde o tratamento e os cuidados são desenvolvidos no ambiente hospitalar, o indivíduo 
tratado isoladamente do convívio cotidiano e a família torna-se destituída de qualquer papel no processo de cuidar (Brasil, 2007).

De acordo com Brasil (2007), a história da instituição hospitalar é marcada por um viés autoritário nas práticas de gestores e trabalhadores, enquadrados por normas e procedimentos regidos em relação ao acompanhante e a visita dos pacientes. Tais normas demandam um novo pensar, em que passa a ser reconhecido o direito do acompanhante e a necessidade de tornar a visita aberta no ambiente hospitalar.

Para Nunes, Silva, Alves e Martins (2009), uma nova visão de saúde da família emerge na tentativa de ultrapassar as fronteiras hospitalares, repercutindo na necessidade de uma assistência humanizada e ética. É essencial a integração da família no cuidado, onde se faz cumprir com os princípios doutrinários da assistência ao usuário do S.U.S: equidade, integralidade e universalidade.

Vários estudos enfatizam a importância da presença do familiar junto ao paciente durante o seu período de internação, principalmente quando se trata de assistência a crianças, pois consideram que a presença do convívio familiar em geral ajuda na recuperação clínica do paciente. Segundo Nunes, Silva, Alves e Martins (2009), a presença do acompanhante nas unidades hospitalares foi regulamentada em 2003 pelo artigo $12^{\circ}$ do Estatuto da Criança e do Adolescente (ECA) e pelo artigo $16^{\circ}$ do Estatuto do Idoso, que atuam garantindo esse direito, e determinam como responsabilidade da instituição propiciar condições adequadas para a permanência do acompanhante por tempo integral, ou seja, até a determinação da alta hospitalar.

Corroborando com Nunes, Silva, Alves e Martins (2009), o Ministério da Saúde (BRASIL, 2007), afirma que também é uma conquista o direito a presença de um acompanhante durante o parto, este regulamentado pela Lei $\mathrm{n}^{\circ} 11.108$, sancionada em 7 de abril de 2005. Esta estabelece o desenvolvimento de profissionais da maternidade ao ajuste do olhar para esse novo integrante, no qual surge associado a ele a necessidade de redimensionamento do espaço físico, que possibilite sua permanência no ambiente hospitalar e a uma abordagem da equipe de Enfermagem no sentido de potencializar a sua presença.

Lautert, Echer e Unicovsky (1998), conceituam o acompanhante como todo e qualquer indivíduo que receba ou não remuneração e que permaneça ao lado do paciente por período de tempo consecutivo e sistemático, proporcionando companhia, apoio emocional, e que execute cuidados sob a orientação e supervisão da equipe de Enfermagem.

Nunes, Silva, Alves e Martins (2009), afirmam que com o crescimento na ênfase familiar no contexto da saúde, observa-se que surge a necessidade de uma reflexão acerca da participação da equipe de Enfermagem sobre o cliente e principalmente o acompanhante, seja ele membro da família, vizinho ou amigo, que ao acompanhar o paciente passa a vivenciar todo processo de hospitalização.

O desenvolvimento desse estudo tem como foco principal conhecer a percepção do profissional Enfermeiro, quanto à permanência do acompanhante do usuário do serviço de saúde, garantido por meio do S.U.S. na unidade hospitalar durante a internação do familiar. 
O interesse em desenvolver o estudo com esta temática surgiu após ler o "Humaniza SUS" documento do Ministério da Saúde o qual tem como objetivo propor o direito ao usuário do S.U.S. de ter um acompanhante por tempo integral durante o período de internação. Por acreditar que a presença do acompanhante, ajuda a um melhor resultado no tratamento do paciente, reduzindo o seu tempo de internação e consequentemente os custos, considera-se ainda importante que a instituição de saúde proporcione um ambiente com condições ideais para garantir a permanência destes.

Ao observar que as unidades de atendimento hospitalares da região do Cariri, com foco na cidade do Crato, local onde foi desenvolvido o estudo, estabelecem um período de tempo para visita dos pacientes que são usuários do S.U.S e que a maioria destes não tem acompanhante por não estarem enquadrados nos casos em que se determina necessária a presença do acompanhante (crianças, parturientes e idosos), surgiu a necessidade de conhecer a percepção que o Enfermeiro tem sobre a presença do acompanhante nas unidades hospitalares, já que com a implantação da proposta do Humaniza SUS, estes estarão mais presentes nos serviços de saúde.

\section{Método}

O presente estudo caracterizou-se por percorrer a trajetória metodológica da pesquisa qualitativa, com abordagem descritiva. Este estudo apresenta-se qualitativo, pois "não é o estudo do fenômeno em si que interessa (...), é na verdade a significação que tal fenômeno ganha para os que o vivenciam" (TURATO, 2005, p.509).

\section{Sujeitos e local do estudo}

Este estudo foi realizado com os Enfermeiros de uma instituição hospitalar de caráter filantrópico, localizada na cidade do Crato-Ceará.

O hospital é constituído por uma equipe multiprofissional, na qual podemos destacar a equipe de Enfermeiros, atualmente formada por 8 profissionais, sendo 7 do sexo feminino e 1 do sexo masculino.

\section{Critérios de Inclusão e Exclusão dos sujeitos do estudo}

Inclusão: a) Ser Enfermeiro da instituição; b) Trabalhar com assistência direta de Enfermagem aos usuários do S.U.S.; c) Anuência para participar do estudo (Leitura e assinatura do Termo de Consentimento Livre e Esclarecido).

Exclusão: a) Ser estagiários, acadêmicos e residentes de Enfermagem. 
Para iniciar o desenvolvimento do estudo foi considerado o cronograma da pesquisa, determinando o período para realização da coleta de dados, e os critérios de inclusão e exclusão para participar da pesquisa. Dentre os 8 profissionais enfermeiros, 4 apresentaram anuência para participar do estudo, os demais profissionais não participaram porque estavam de férias ( 2 enfermeiros/as), não presta assistência a pacientes do S.U.S (1 enfermeiro/a) e não aceitou participar do estudo (1 enfermeiro/a).

Para a coleta de dados foi utilizado um formulário semi-estruturado, em que constavam aspectos do perfil profissional dos entrevistados, e perguntas abertas direcionadas à abordagem das questõesproblemas do estudo. O formulário segundo Carvalho (2003, p.156), consiste numa "(...) coleção de questões que são perguntadas e anotadas por um entrevistador, numa situação 'face a face".

Após aceitação para participar do estudo e assinatura do Termo de Consentimento Livre e Esclarecido os profissionais responderam um formulário semi-estruturado constituído por dois blocos, no qual se buscou conhecer no primeiro bloco os aspectos relacionados ao perfil dos sujeitos do estudo e o bloco 2 com perguntas subjetivas acerca do assunto em foco, que serão abordadas em categorias de estudo na seguinte seqüência: "Concepção do que é ser acompanhante"; "Importância da presença de um familiar durante a hospitalização"; "Existência de conflito entre equipe de saúde e acompanhante"; "Acompanhante influencia no quadro clínico do paciente"; "Tempo suficiente para que o acompanhante permaneça junto ao familiar durante a hospitalização".

Para efetivar a coleta de dados, foi solicitado primeiramente à Coordenação do Curso de Especialização em Enfermagem Clínica Médica-Cirúrgica, localizada na cidade de Crato - CE, um ofício, visando à solicitação da concessão de campo ao responsável pela instituição em que atuam os Enfermeiros do estudo.

Foi feita uma abordagem dos Enfermeiros durante o período de 1 (uma) semana, mais precisamente nos dias 5 a 12 do mês janeiro de 2011.

Todos os Enfermeiros, que trabalham com assistência direta aos usuários do S.U.S. foram abordados nos seus setores de trabalho: clínica médica, clínica cirúrgica, centro cirúrgico, pediatria, UTI e maternidade, para fins de esclarecimentos acerca dos objetivos do estudo e solicitação para colaborar no desenvolvimento desta pesquisa, após aceitação para participar do estudo foi entregue a cada profissional o formulário e agendada uma data para o seu recebimento.

\section{Análise e Discussão dos Dados}

O critério de saturação dos dados foi o considerado nesta pesquisa. Foram apresentados os resultados em "categorias de estudo", emergidas do campo, e de acordo com os objetivos do trabalho, 
sempre comparando os resultados encontrados com a literatura científica acerca da temática em estudo.

De acordo com Fonseca Júnior (2005), "a categorização consiste no trabalho de classificação e reagrupamento das unidades de registro em número reduzido de categorias, com o objetivo de torna inteligível a massa de dados e sua diversidade".

Com relação aos aspectos éticos, foi levada em consideração a prática preconizada no Brasil em 1996, por meio da Resolução 196/96, que trata da pesquisa envolvendo seres humanos (BRASIL, 1996).

Em conformidade com a resolução supracitada, ressaltamos que em hipótese alguma foi divulgado o nome das pessoas envolvidas. Para garantia do anonimato dos, participantes estes foram identificados como: "Entrevistado 1"; "Entrevistado 2" e assim por diante.

\section{Resultados e Discussão}

O estudo foi realizado em um hospital de caráter filantrópico na cidade do Crato-Ceará. O campo de pesquisa deste estudo foi escolhido por ter sido a instituição na qual a pesquisadora desenvolveu seus estágios referentes às práticas da especialização em Clínica Médico-Cirurgica.

Após a análise do bloco, que caracteriza os sujeitos participantes do estudo, pôde-se observar que são profissionais que embora atuem em setores como emergência; UTI; centro cirúrgico; pediatria; clínica médica; clínica cirúrgica; maternidade, em sua maioria apresentam experiência de 1 a 3 anos em clínica médico-cirurgica.

75\% destes profissionais não trabalham em outra instituição de saúde, e todos trabalham cumprindo uma carga horária de 8 horas de trabalho diário e perfazendo 40 horas semanais, na instituição da pesquisa.

\section{Concepção do que é ser acompanhante}

É primordial conhecer a concepção que os profissionais da saúde atribuem ao termo "acompanhante", já que sempre que se tem um familiar/ amigo/ conhecido doente alguém assume o papel de acompanhante ou visitante, sabendo que este papel também pode ser desenvolvido pelo próprio profissional da saúde, quando não está no período do seu exercício profissional.

Os enfermeiros caracterizaram o acompanhante como sendo alguém que dê apoio, que seja capaz de colaborar e auxiliar os profissionais da saúde na execução de cuidados básicos ao paciente. Conforme pode evidenciar nos registro abaixo:

"Um aliado para a recuperação do paciente" (Entrevistado 1). 
"É o indivíduo "capaz" de estar ao lado do cliente para auxiliar o cliente e equipe em atividades simples" (Entrevistado 3).

"É prestar apoio psicoemocional, ajudar nos cuidados de higiene pessoal e colaborar com a equipe do hospital nos cuidados do paciente" (Entrevistado 4).

Embora o estudo realizado mostre pontos positivos quanto ao que é ser acompanhante, estudos desenvolvidos por Soares e Leventhal (2008), mostram que a equipe de enfermagem deve ser trabalhada para aceitar a permanência desse novo membro na unidade hospitalar, o acompanhante, já que a sua presença torna-se indispensável para o tratamento e recuperação do familiar.

A partir de vários estudos começaram a perceber que a família pode contribuir e interagir com a equipe de saúde favorecendo no seu tratamento e influenciando na recuperação emocional do paciente. Essa interação vem sendo frequente, devido ao maior conhecimento das pessoas sobre os seus direitos (LUZ, MELNIK, BERNARDINO e OLIVEIRA, 2009).

A presença do familiar ou amigo é um elemento de extrema importância para recuperação do cliente, independente do comprometimento físico. Um dos maiores problemas enfrentados pelos pacientes internados em Centro de Terapia Intensiva é a separação da família, sem dúvida a presença desta na hospitalização reduz a ansiedade e favorece a recuperação além de cooperar na implantação dos cuidados facilitando a resolução dos problemas (LACERDA, CARVALHO e ROCHA, 2004).

\section{Importância da presença de um familiar durante a hospitalização}

Embora saibamos que sempre que surge um processo de internação uma das manifestações mais comum dos familiares é a de querer acompanhar o seu ente durante o processo de hospitalização, já que este estará exposto a um novo ambiente, realização de procedimentos e cuidados anteriormente não realizados, o que mostra a necessidade do enfermeiro reconhecer a importância do familiar nesse processo.

A partir da análise das respostas dos entrevistados, pode-se observar que o acompanhante auxilia positivamente durante a anamnese do paciente, relatando fatos que o mesmo não lembra, além de servir como testemunha para as condutas médicas adotadas, e como ponto negativo, a presença deste poder atuar dificultando o tratamento do cliente, como se pode observar pelos relatos:

"Sim. Pois o mesmo detém informações importantes que até mesmo o paciente pode não saber" (Entrevistado 1).

"Depende. Porque infelizmente alguns acompanhantes e/ou familiares atrapalham a terapia do cliente" (Entrevistado 2).

"Sim, para que ajude na coleta de informações da anamnese, para que sirva de testemunha da conduta médica adotada, para prestar apoio psicoemocional e para que seja mais uma pessoa a ser orientada quanto aos cuidados com o paciente" (Entrevistado 4). 
As relações entre as pessoas que interagem no ambiente de internação hospitalar não acontecem de forma linear, mesmo que os sujeitos participantes sejam constantes, pois vários fatores, positivos ou negativos, contribuem para o desenvolvimento da relação enfermeiro, paciente e acompanhante. Há situações em que a equipe de enfermagem manifesta seu poder, e em outras, solidariedade e sensibilidade para com a realização do cuidado e o acompanhante pode atuar dificultando o desenvolvimento de atividades a partir de atitudes não cooperativas, não participativa e desatenciosa com a equipe ou com o seu próprio familiar (SQVASSANTE e ALVIM, 2009).

O familiar é considerado uma fonte de segurança e carinho, e a importância deste é enfatizada pelo fato dele conseguir perceber as alterações na saúde do familiar, tornando-se muitas das vezes o responsável por informar as alterações apresentadas na saúde destes. Diante disto, o familiar não pode ser percebido apenas como um receptáculo e provedor de informações é importante que ele possa participar ativamente do processo terapêutico dentro de suas limitações (QUIRINO, COLLET e NEVES, 2010).

O cuidador/ familiar deve fazer parte do foco da assistência prestada ao cliente, atendendo aos preceitos de um atendimento humanista e de forma holística no contexto da família. É fundamental compreender e aceitar que as famílias envolvidas no tratamento e cura têm seus próprios conceitos sobre saúde, doença e cuidado e não podem ser ignorados pelas enfermeiras (PAI e SOARES, 1999).

A presença do acompanhante/familiar durante o processo da hospitalização traz consigo alterações nas relações de trabalho, pois a introdução deste no cuidado precisa ainda ser bem compreendida pela equipe, as negociações com o cuidado a ser prestado precisa ainda ser bem definida para os profissionais e familiares (SOARES e LEVENTHAL, 2008).

A necessidade de um diálogo entre a equipe de enfermagem e acompanhante é fundamental para que se possa ser determinado o que ele pode fazer e o que é de competência de cada um. A forma de se conduzir a relação com o familiar no cuidado ao cliente hospitalizado deve ser coerente com a atual política de humanização do atendimento à saúde, pois estes devem centrar nos princípios da integralidade, equidade e participação dos usuários na assistência, passando a desenvolver práticas em que seja valorizada a participação de todos os envolvidos no processo e conseqüentemente favorecerá ao processo de recuperação do cliente (SQVASSANTE e ALVIM, 2009).

\section{Existência de conflito entre a equipe de saúde e acompanhante}

O humaniza SUS surge com o objetivo de integrar o acompanhante a todos os pacientes internados durante todo o período de hospitalização do usuário do SUS. Para tanto se faz necessário 
conhecer como se encontra a relação entre equipe de saúde e acompanhante, se existem ou não conflitos quando da interação destes.

De acordo com os enfermeiros entrevistados os conflitos podem ser decorrentes da existência de normas e rotinas que muitas vezes não são de conhecimento dos acompanhantes, ou pela falta de compreensão em relação ao seu papel como "acompanhante" ou por desconhecerem o porquê de algumas condutas médicas adotadas. Então por meio dos registros dos profissionais sobre a existência de conflitos entre a equipe de enfermagem e acompanhante pode-se observar:

"Sim. Pois o acompanhante por muitas vezes não entende ou até mesmo não quer entender a real situação do paciente" (Entrevistado 1).

"Não diria conflito, mas falta a definição de atividades do acompanhante que muitas vezes é ausente do cliente" (Entrevistado 3).

"O conflito sé existe quando não há uma conversa clara entre ambos e um bom entendimento. O fator emocional conta muito e interfere na absorção das informações. Muitas vezes eles não entendem o "porque" da conduta médica adotada e isso gera conflito. As normas e rotinas da instituição também não são bem aceitas a menos que o acompanhante entenda a necessidade dessas normas. As diferenças culturais também interferem nessa relação" (Entrevistado 4).

A presença de um familiar imprime a necessidade de uma nova forma de organização no trabalho da enfermagem, uma vez que muitos cuidados passam a ser delegados para esse novo integrante. A falta de uma política institucional que defina claramente o papel do acompanhante faz com que os profissionais da saúde determinem o que eles devem fazer, e por consequiência do desconhecimento dos acompanhantes acerca dos seus direitos quanto a sua permanência durante o período de hospitalização faz com que eles passem a desenvolver atividades delegadas pelos profissionais por medo de represálias quanto a sua permanência no ambiente hospitalar (SQVASSANTE e ALVIM, 2009).

Em um estudo realizado por Soares e Leventhal (2008), observou que a maioria dos familiares relatam receberem informações insuficientes a respeito do atendimento a criança/familiar, bem, como do papel que devem desempenhar no hospital e dos procedimentos diagnósticos e terapêuticos decorrentes da doença e hospitalização, assim como seus direitos, deveres e participação nos cuidados. É importante salientar que a orientação quanto às normas e rotinas hospitalares é uma das maneiras da equipe de enfermagem criar o primeiro vínculo com o paciente e família, além de ser uma forma de reduzir a ansiedade e evitar conflitos pelo desconhecimento destes com a equipe de enfermagem acerca dos protocolos institucionais.

Muitas vezes os atritos criados dentro das instituições hospitalares devem-se pelo fato do processo de hospitalização de um familiar se caracterizar por um período de mudanças, onde o 
familiar e doente se deparam com um ambiente estranho, com horários determinados, protocolos institucionais e pessoas que ora informam o que deve, pode ou não fazer, o que gera insatisfação e conseqüentemente conflitos com a equipe de enfermagem, equipe que permanece ininterruptamente no hospital e de mais fácil acesso para o familiar (SQVASSANTE e ALVIM, 2009).

Embora Quirino, Collet e Neves (2010), considerem de forma positiva uma relação de cooperação entre os profissionais e familiares, infelizmente ainda é muito comum a predominância de uma relação de dominação-subordinação. O que acontece devido os profissionais quererem transformar os direitos da família em acompanhar o paciente/criança, em um dever, assumindo o seu papel em acompanhá-las e desenvolvendo atividades que não são de sua competência e sim dos profissionais do serviço. E quando os acompanhantes desenvolvem qualquer comportamento contrário ao esperado pelos profissionais ocorrem conflitos e distanciamento, repercutindo na assistência prestada ao paciente, onde os profissionais passam a ignorar ou negligenciar as necessidades do cliente internado.

Para tanto é importante que no primeiro contato com o acompanhante sejam informados as normas e rotinas da instituição, bem como identificar se estas foram realmente compreendidas, pois muitas vezes o silêncio apresentado pelo acompanhante após receber todas as informações pode indicar uma dificuldade na sua compreensão. É necessário que sejam definidas as ações de responsabilidade de cada sujeito, equipe/acompanhante. Infelizmente o que se observa nas unidades de saúde é que alguns profissionais no momento de promover a educação e informações em saúde limitam-se apenas a dizer o que eles consideram necessário (SQVASSANTE e ALVIM, 2009).

\section{Acompanhante influencia no quadro clínico do paciente}

A presença do acompanhante é permitida por tempo integral para as crianças, idosos e pacientes que tenham dependência total para realização das atividades. Estudos mostram que sempre que o paciente hospitalizado tem a presença de um acompanhante durante a internação, mais rápido é seu processo de recuperação.

Quando interrogamos os enfermeiros se eles acham que a presença do acompanhante influência no quadro clínico do paciente, eles relatam que quando estes estão presentes passam maior segurança e ajudam no tratamento, mas que para que essa contribuição seja positiva é necessário que haja uma boa interação entre acompanhante e paciente, pois caso contrário ele poderá atuar retardando o processo de recuperação, como apresentados nos relatos:

"Sim. A presença de alguém conhecido transmite segurança ao paciente e o ajuda no tratamento para tomada de decisões" (Entrevistado 1).

"Sim. Pois muitos sentem-se mais confiante, e podem ter um incentivos maior para sua recuperação. E ao contrário, quando o acompanhante não tem perfil (nervoso, assusta-se com tudo e passa para o 
cliente; é agressivo...) Isso só piora o seu psicológico e consequentemente o fisiológico" (Entrevistado3).

"Sim. Pode influenciar negativamente ou positivamente dependendo do relacionamento entre eles. $\mathrm{O}$ acompanhante tem que passar tranqüilidade para o paciente, e não deixá-lo mais estressado" (Entrevistado 4).

A hospitalização é uma experiência em que os acompanhantes lidam com a difícil realidade, movidos pela crença de que o indivíduo doente irá melhorar e por isso se mostram dispostos a estarem com o outro, seja qual for a situação. Quando é permitida a sua presença durante a internação de estar junto ao doente, os familiares podem exercer o seu papel solidário, favorecendo ao processo interacional onde a reciprocidade de sentimentos promove um relacionamento benéfico tanto para o doente como para o familiar capaz de aliviar o sentimento de ambos (SILVA, BOCCHI e BOUSSO, 2008).

A presença do acompanhante proporciona maior conforto e qualidade de vida ao cliente, além de favorecer a uma melhor abordagem da enfermagem. Embora a presença do acompanhante não seja regulamentada ainda para todos os indivíduos, ela necessita ser absorvida pelos profissionais, já que a participação da família no planejamento dos cuidados favorece a uma melhor identificação das necessidades do indivíduo, possibilitando um cuidado mais humanizado, holístico e integral (QUIRINO, COLLET e NEVES, 2010).

A valorização da presença materna/familiar e um processo educativo e informativo de mão dupla entre acompanhante e equipe são considerados a base para o desenvolvimento de uma assistencial humanizada (FAQUINELLO, HIGANASHI e MARCON, 2007).

\section{Tempo necessário para que o acompanhante permaneça junto ao familiar durante a hospitalização}

Foram entrevistados enfermeiros de diferentes áreas de atendimento na assistência, como clínica médica, clinica cirúrgica, centro cirúrgico, UTI, emergência, maternidade, locais estes em que sempre se tem a presença e/ou necessidade de um acompanhante para estar junto ao cliente. Para tanto surge à necessidade de conhecer quanto tempo os enfermeiros consideram necessários para que o acompanhante permaneça junto ao paciente.

Baseados nesses questionamentos muitos desses profissionais afirmam que a presença deste deve ser até que o paciente passe a desenvolver suas atividades de forma independente.

"Depende do estado do cliente até que seja dependente de cuidados, ou de acordo com a necessidade" (Entrevistado 2).

"Até a estabilização clínica. Se for idoso, criança ou deficiente durante toda hospitalização" (Entrevistado 3). 
Para Lacerda, Carvalho e Rocha (2004), a presença do acompanhante proporciona um maior conforto, qualidade de vida e estimula a recuperação do cliente internado em uma CTI (Centro de Terapia Intensiva), para tanto é importante envolver por um maior período a família no planejamento das ações de enfermagem, pois a sua cooperação irá auxiliar na implementação dos cuidados junto ao cliente.

Silva, Bocchi e Bousso (2008), enfatizam o artigo 26 dos Direitos dos Pacientes onde é garantido, o direito a presença do acompanhante, quando se desejar, durante as consultas e internações, porém as visitas devem ser disciplinadas em horários compatíveis, de forma que não comprometam as atividades médico-sanitárias. Aos idosos é garantido o direito a ter o acompanhante por tempo integral, devendo ser garantida boas condições na instituição para a sua permanência, existem casos em que a permanência deste não é regulamentada em lei, como no caso dos adultos, no qual a determinação da presença deste fica a critério do enfermeiro da instituição a partir da avaliação do grau de dependência do paciente, ou seja da capacidade do cliente em desenvolver seu próprio auto-cuidado, e quando ocorre a permissão para a permanência destes tem-se um alívio no sofrimento do cliente e familiar.

A presença do acompanhante é um direito do paciente (idoso, criança e parturiente), porém os profissionais da saúde precisam se adaptar a essa evolução e aceitar a participação deste no ambiente hospitalar, buscando interagir com o familiar ao invés de criar barreiras, justificando que estes interferem na dinâmica do serviço (SOARES e LEVENTHAL, 2008).

O familiar/acompanhante é o que permanece mais tempo com o cliente, passando mais tempo dentro da instituição e vivenciando a cultura deste espaço, reconhecendo os profissionais, suas terminologias e muitas vezes passando a reproduzir cuidados que são de responsabilidade dos profissionais, por isso deve ser visto como um indivíduo que também precisa de cuidados e orientações que venham a beneficiar o cuidado do seu familiar, assim como o seu próprio cuidado (SOUZA e OLIVEIRA, 2010).

Assim como enfatizam Maciel e Souza (2006), o acompanhante é uma fonte de conforto e segurança para o cliente, um elo com a equipe e um fator contribuinte para a melhoria da assistência prestada. A permanência deste na unidade hospitalar contribui para humanização, pois o avanço tecnológico transforma o ambiente e as pessoas que nele trabalham, passando a ser um elo entre a equipe, família e pessoa doente.

\section{Referências}


BRASIL, Ministério da Saúde.(1996). Conselho Nacional de Saúde. Comissão Nacional de Ética em pesquisa. Normas para pesquisa envolvendo seres humanos: (Res. CNS 196/96 e outros). Brasília (DF).

Ministério da Saúde. (2007). Humaniza SUS Visita aberta e direito ao acompanhante. $2^{\circ}$ edição. Brasília-DF.

CARVAlHO, M.C.(2003). Construindo o Saber, Metodologia Científica: Fundamentos e Técnica, Papiros, 14ed.

FONSECA JÚNIOR,W.C.da.(2005). Análise do conteúdo. In: Duarte, Jorge, Barros, Antonio. (Org) . Métodos e Técnicas de pesquisa em comunicação. São Paulo: Atlas. P.280-92

LACERDA, A.C.de; CARVALHO, A.C.S.de.; ROCHA, R.M. (2004). Acompanhantes no Centro de Terapia Intensiva: Percepção da equipe de enfermagem. Revista de Enferm UERJ; 12:18-23.

LAUTERT, L.; ECHER, I.C.; UNIOVSKY, M.A.R. (1998). O Acompanhante do paciente adulto hospitalizado. Rev .Gaúcha Enferm. Porto Alegra. V.19, n²,p.118-131, jul.

LUZ,C.F.da; MELNIK, M.G.; BERNARDINO,E.; OLIVEIRA, E.S.de.(2009). Compreendendo as restrições dos técnicos de enfermagem sobre a permanência de acompanhantes em Unidade de Terapia Intensiva Aberta. Texto e Contexto-enferm. vol.18, n² 2 . Florianópolis abr./jun. Disponível em: http://www.scielo.br/pdf/tce/v18n2/14.pdf Acesso:14/01/2011.

MACIEL, M.R.; SOUZA, M.F. (2006). Acompanhante de adulto na Unidade de Terapia Intensiva: uma visão do paciente. Acta Paul Enferm. 19(2):138-43.

NUNES, E.C.D.A., SILVA,K.de J.C., ALVES,R.R., MARTINS, A.de O. (2009). Percepção da equipe de enfermagem acerca dos acompanhantes hospitalares. Revista Nursing, 12(139): 565-569.

PAI, M.M.; SOARES,M.A.L. (1999). Percepção do significado da função do cuidador por um grupo de enfermeiras e cuidadores: convergências e divergências. Rev. Esc.Enf. USP. v.33. n³. p. 231-5. Set.

QUIRINO, D.D.; COLLET, N.; NEVES, A.F.G.B.(2010). Hospitalização Infantil: concepções de enfermagem acerca da mãe acompanhante. Rev. Gaúcha Enferm., Porto Alegre(RS). Jun; 31(2): 3006.

SILVA, L.; BOCCHI, S.C.; BOUSSO, R.S. (2008). O papel da solidariedade desempenhado por familiares visitantes e acompanhante de adultos e idosos hospitalizados. Texto contexto- enferm. v.17 n.2 Florianópolis abr./jun. Disponível em: http://www.scielo.br/pdf/tce/v17n2/11.pdf Acesso: 20/03/2011.

SOARES, M.de F.; LEVENTHAL,L.C.(2008). A relação entre a equipe de enfermagem e o acompanhante da criança hospitalizada: facilidades e dificuldades. Cienc. Cuid. Saúde. Jul/Set; 7(3):327-332.

SOUZA, T.V.; OLIVEIRA, I.C.S.dos.(2010). Interação familiar/acompanhante e equipe de enfermagem no cuidado à criança hospitalizada: perspectivas para a enfermagem pediátrica. Esc. Anna Nery. jul.-set; 14 (3):551-559.

SQUASSANTE, N.D.; ALVIM, N.A.T. (2009). Relação equipe de enfermagem e acompanhantes de clientes hospitalizados: implicações para o cuidado*. Revista Brasileira de Enfermagem. Brasília. Jan-Fev 62(1): 11-7. 
TURATO, E. R. (2005). Métodos qualitativos e quantitativos na área da saúde: definições, diferenças e seus objetos de pesquisa. Rev. Saúde Pública [online]. v. 39, n. 3, pp. 507-514. ISSN 0034-8910

\section{Sobre os autores:}

(1) Juliana Saraiva de Alencar, Enfermeira Graduada pela Faculdade Santa Maria, Pós-graduada em Enfermagem Clínica Médico-Cirurgica pelo Centro Universitário São Camilo; e Enfermagem do Trabalho pela Faculdades Integradas de Patos (FIP). Professora Orientadora de Estágios do Instituto Centro de Ensino Tecnológico do Ceará (CENTEC-CE). Endereço: Rua Cícero Araripe, 245 Pimenta, Crato-Ce. E-mail: julianaaa100@ yahoo.com.br;

Continua...

(2) Lorena Saraiva de Alencar, Acadêmica de Medicina do $10^{\circ}$ Período da Universidade Federal do Ceará (UFC Campus Barbalha/Ce), Endereço: Rua Cícero Araripe, 245 Pimenta, Crato-Ce. E-mail: loreninhaloris@hotmail.com;

(3) Patrícia Luciany Almeida Macêdo da Silva, Enfermeira Graduada pela Faculdade Leão Sampaio, Especialista em Urgência e Emergência pela Universidade Vale do Acarau - UVA, Especialista em Saúde Pública pela Universidade Estadual do Ceará -UECE. Endereço: Rua Ailton Gomes 841, Franciscanos, Juazeiro do Norte-CE.

(4) Josberto Calixto Pereira, Enfermeiro Graduado pela Faculdade Santa Maria, Especialista em Urgência e Emergência pela Faculdades Integradas de Patos - FIP, Especialista em Unidade de Terapia Intensiva pela Faculdade Leão Sampaio, Especialista em Docencia do Ensino Superior e Educação Continuada pela Faculdade de Juazeiro do Norte - FJN. Rua São Benedito 1527, Franciscanos, Juazeiro do Norte$\mathrm{CE}$

\section{Como citar este artigo (Formato ISO):}

ALENCAR, J.S.; ALENCAR L.S.; SILVA, P.L.A.M. e PEREIRA, J.C.. Percepção dos Enfermeiros quanto à participação do acompanhante durante a internação hospitalar de um usuário do Sistema Único de Saúde (SUS). Id on Line Revista de Psicologia, Novembro de 2012, vol.1, n.18, p.33-45. ISSN 1981-1189. 